\title{
Efecto de las propiedades físicas del carbón activado en la adsorción de oro desde medio cianuro ${ }^{(\bullet)}$
}

\author{
P. Navarro* y C. Vargas*
}

\begin{abstract}
Resumen
Se estudió el efecto de las propiedades físicas de un carbón activado, tales como distribución de tamaño de poros, superficie específica, diámetro promedio de poros, en la adsorción de oro desde disoluciones cianuradas, con el oro disuelto a la forma de $\mathrm{Au}(\mathrm{CN})_{2}^{-}$. Para cumplir con los objetivos planteados se trabajó con dos carbones activados: el carbón A, con superficie específica de $985 \mathrm{~m}^{2} / \mathrm{g}, 57 \%$ de microporos y $3,8 \mathrm{~nm}$ como diámetro promedio de poros, y el carbón B, con superficie específica de $786 \mathrm{~m}^{2} / \mathrm{g}, 27 \%$ de microporos y 11,6 nm como diámetro promedio de poros; ambos carbones, de forma granular, han sido fabricados a partir de cáscara de coco. Se realizaron ensayos de adsorción batch en un reactor de $500 \mathrm{ml}$ de capacidad, con agitación mecánica a temperatura constante. Se estudiaron los efectos de cationes presentes, tales como $\mathrm{Ca}^{2+}, \mathrm{Na}^{+}, \mathrm{K}^{+}$y $\mathrm{Li}^{+}$, de la distribución de tamaño de poros, del diámetro promedio de poros y del área superficial, en la velocidad y cantidad de oro adsorbido en los carbones activados denominados como A y B. Se encontró que las propiedades físicas de un carbón activado constituyen un factor relevante en el proceso de adsorción de oro, en términos de velocidad y cantidad de oro adsorbido. El carbón B, con $786 \mathrm{~m}^{2} / \mathrm{g}$ de superficie específica, alcanzó una mayor carga por unidad de superficie $\left(0,02 \mathrm{mg} \mathrm{Au} / \mathrm{m}^{2}\right)$ en relación al carbón A, de $985 \mathrm{~m}^{2} / \mathrm{g}$, el cual alcanzó una carga de $0,01 \mathrm{mg} \mathrm{Au} / \mathrm{m}^{2}$, después de 6 h de contacto carbón-disolución. La velocidad de adsorción de oro en ambos carbones fue controlada por transferencia de masa en la película líquida que rodea a las partículas de carbón, a tiempos cortos o cargas pequeñas de oro en las partículas, lejos del equilibrio. Al aplicar un modelo cinético de primer orden, se obtuvo que la razón de las constantes cinéticas para los carbones A y B, es decir, $\left(\mathrm{k}_{\mathrm{B}} / \mathrm{k}_{\mathrm{A}}\right)$, fluctúa en un valor de 3 para los distintos cationes en estudio. En general, es posible afirmar que la velocidad de adsorción y la cantidad de oro adsorbido aumentan con el aumento de macroporos y con el aumento del diámetro promedio de poros. La presencia de cationes favorece el proceso de adsorción de oro en medio cianuro, especialmente los de mayor valencia.
\end{abstract}

Palabras clave Propiedades físicas; Carbón activado; Adsorción; Oro.

\section{Effect of the physical properites of activated carbon in the gold adsorption from cyanide media}

\begin{abstract}
The effect of the physical properties of an activated carbon such as pore size distribution, specific surface, pore average diameter, in the gold adsorption from cyanide solution with the gold to the $\mathrm{Au}(\mathrm{CN})_{2}^{-}$form, was studied. To meet the proposed objectives two carbons were studied: carbon A with specific surface of $985 \mathrm{~m}^{2} / \mathrm{g}, 57 \%$ of micropores and $1.85 \mathrm{~nm}$ as average diameter of pores and carbon B with specific surface of $786 \mathrm{~m}^{2} / \mathrm{g}, 27 \%$ and pores of $2.35 \mathrm{~nm}$ as average diameter of pores; both granular carbons made from coconut shell. Batch adsorption tests were performed in a reactor of $500 \mathrm{ml}$ of capacity with mechanical stirring at constant temperature. The effect of cations present in the aqueous solutions such as $\mathrm{Ca}^{2+}, \mathrm{Na}^{+}, \mathrm{K}+$ and $\mathrm{Li}^{+}$, the effect of pore size distribution, the effect of average pore diameter and surface area were evaluated in function of the rate and amount of gold adsorbed on the activated carbons denominated as A and B. The results to indicate that the physical properties of an activated carbon are an important factor in the gold adsorption process in terms of rate and amount of adsorbed gold. The carbon B with $786 \mathrm{~m}^{2} / \mathrm{g}$ of specific surface area reached a higher load per unit area $\left(0.02 \mathrm{mg} \mathrm{Au} / \mathrm{m}^{2}\right)$ in relation to the carbon B of $985 \mathrm{~m}^{2} / \mathrm{g}$ which had a load of $0.01 \mathrm{mg} \mathrm{Au} / \mathrm{m}^{2}$, after $6 \mathrm{~h}$ of contact carbon-solution. The rate adsorption of gold in both carbons is controlled by mass transfer in the liquid film surrounding the carbon particles to short times or small loads of gold in the particles, far from equilibrium. Applying a first order kinetic model, it was obtained that the ratio of the kinetic constants for carbons $A$ and $B$, ie $\left(k_{B} / k_{A}\right)$, fluctuates in a value of 3 for the different cations in study. In general it is possible to say that the rate adsorption and the amount of adsorbed gold increased with the increase in macropores and with the increasing pore average diameter. The presence of cations favors the gold adsorption in cyanide media, especially those of higher valence.
\end{abstract}




\section{INTRODUCCIÓN}

El uso del carbón activado se ha incrementado en el campo de la Hidrometalurgia, especialmente, en la recuperación de oro y plata desde soluciones cianuradas, por ser el más versátil de los adsorbentes sólidos usados en la industria, debido a su elevada área superficial y su distribución de tamaño de poro, bimodal (a veces trimodal), que proporciona el buen acceso de las moléculas del adsorbato al interior de las partículas $^{[1-3]}$.

Carbón activado es un término genérico que designa a una familia de materiales carbonáceos, altamente porosos, que no pueden ser caracterizados por una fórmula estructural o por análisis químico. Los poros son cavidades pequeñas en las partículas de carbón, que acogen en sus paredes internas agentes moleculares contenidos en fluidos que rodean las partículas. En esta forma, actúa como una malla molecular que posee una alta especificidad y logra separar especies muy definidas molecularmente. El volumen de los poros en carbones activados es, habitualmente, definido como mayor que $0,2 \mathrm{ml} / \mathrm{g}$ y el área superficial interna es, generalmente, entre 400 a 1.500 $\mathrm{m}^{2} / \mathrm{g}$. Esta área es bastante grande, si se tiene en cuenta que la superficie específica de una esfera de carbón sin porosidad alguna es alrededor de $3,86 \cdot 10^{-4}$ $\mathrm{m}^{2} / \mathrm{g}$. Estudios de difracción de rayos X han demostrado que la estructura del carbón activado es similar a la del grafito. El grafito ideal consta de átomos de carbono enlazados covalentemente en los planos hexagonales de su estructura y enlazados entre planos por débiles fuerzas de Van der Waals ${ }^{[4}$ y 5$]$.

Hoy en día, numerosas plantas de cianuración de oro aplican la tecnología del carbón activado, gracias a las ventajas competitivas que ofrece respecto a la técnica de cementación, siendo en la actualidad el principal proceso industrial utilizado para recuperar metales de interés desde soluciones acuosas. Además, el creciente interés de diversas compañías por la explotación de yacimientos de oro se origina por dos motivos: el aumento en el precio del oro y la creación de métodos de operación cada vez más rentables para la explotación de yacimientos de baja ley, que antes no lo eran.

A pesar de las amplias aplicaciones industriales de esta tecnología, el mecanismo de adsorción de complejos cianometálicos en carbón activado, incluyendo oro, no está completamente comprendido. Desde un punto de vista mecanístico, diversos autores han publicado que el dicianuro de oro es adsorbido, sin ningún cambio químico, desde soluciones alcalinas dentro del carbón activado, involucrando el mecanismo de par iónico del tipo $\left[\mathrm{M}^{+\mathrm{n}}\right]\left[\mathrm{Au}(\mathrm{CN})_{2}^{-}\right]_{\mathrm{n}}$ en la superficie del carbón, donde $\mathrm{M}^{+\mathrm{n}}$ representa un tipo de cationes como $\mathrm{Na}^{+}, \mathrm{K}+, \mathrm{o} \mathrm{Ca}^{+2}$, frecuentes en los sistemas químicos, e identificaron factores que afectan el proceso, dentro de los cuales se encuentran el efecto de la fuerza iónica y la temperatura.

En las décadas pasadas se ha realizado gran cantidad de investigación acerca de la adsorción de especies de aurocianuro en carbones activados. Davidson ${ }^{[6]}$ propuso que el oro es adsorbido involucrando el mecanismo de par iónico del tipo $\mathrm{Mn}^{+}\left[\mathrm{Au}(\mathrm{CN})_{2}^{-}\right]_{n}$ en la superficie del carbón, donde $\mathrm{Mn}+$ representa un tipo de catión como $\mathrm{Na}^{+}, \mathrm{K}^{+}, \mathrm{o} \mathrm{Ca}^{+2}$. Este mecanismo propuesto ha sido ampliamente aceptado en estudios de adsorción de oro. Mc Dougall et al. ${ }^{[7]}$, utilizando espectroscopía de Rayos X, mostraron que la adsorción de aurocianuro en presencia o ausencia de electrolitos y ácidos se realiza por mecanismos iguales. Aunque la identidad del aurocianuro adsorbido no se conoce, el mecanismo tendría una etapa inicial de adsorción, que involucra la adsorción de un par iónico, donde $\mathrm{M}^{\mathrm{n}+}$ es un ión metálico que tiene una solubilidad más baja que el $\mathrm{Au}(\mathrm{CN})_{2}^{-}$en el medio de adsorción, seguida por una etapa de reducción, en la cual se forman especies del tipo $\mathrm{Au}(\mathrm{CN})_{\mathrm{x}}$ en la superficie del carbón. Mc Dougall y Hancock ${ }^{[4]}$ y, en forma más reciente, López-Ramón et al ${ }^{[8]}$, confirmaron el mecanismo anteriormente propuesto y concluyeron que el mecanismo más probable de carga del oro bajo condiciones típicas de operación CIP involucra la adsorción de aurocianuro sin cambio químico. Adams y Fleming ${ }^{[9]}$, encontraron que bajo condiciones alcalinas típicas de un proceso CIP, el oro es extraído como un par iónico. A bajos valores de $\mathrm{pH}$ $(<3)$, el oro es cargado en el carbón, predominantemente, como ácido aurocianuro, $\mathrm{HAu}(\mathrm{CN})_{2}$, mientras que a $\mathrm{pH}$ intermedios, el par iónico y el ácido son cargados simultáneamente. Cuando el oro era cargado en el carbón a bajos valores de $\mathrm{pH}$, algo de oro era descompuesto a $\mathrm{AuCN}$, pero la cantidad descompuesta era significativa solo a cargas muy altas. Adams, M.D. ${ }^{[10]}$ plantea que el desacuerdo entre diferentes investigadores con respecto al mecanismo exacto involucrado en la adsorción de aurocianuro en carbón activado es debido a diferencias en las condiciones experimentales que ellos han utilizado. Así, por ejemplo, las investigaciones fueron realizadas con una variedad de carbones, preparados desde un rango de materias primas bajo diferentes condiciones experimentales, en términos de la concentración de oro en disolución y la presencia (o ausencia) de varios tipos de aditivos en el medio de carga. Los efectos del oxígeno y la fuerza iónica estarían involucrados. La adsorción bajo condiciones de alta fuerza iónica, como en plantas reales, involucra un mecanismo de par iónico en el cual los pares iónicos del tipo $\mathrm{M}^{\mathrm{n}+}\left[\mathrm{Au}(\mathrm{CN})_{2}^{-}\right] \mathrm{n}$ son adsorbidos en la superficie del carbón sin cambio 
químico y, bajo estas condiciones, el oxígeno tiene un rol insignificante; mientras que bajo condiciones de baja fuerza iónica, una porción importante del oro es adsorbido por interacción electrostática con sitios de intercambio iónico formados por oxidación de la superficie del carbón por oxígeno molecular y una porción menor lo es por par iónico en ausencia de oxígeno.

Claramente, la literatura sugiere que la adsorción de especies del tipo $\mathrm{M}(\mathrm{CN})_{2}^{-}$en carbón activado puede estar influenciada por un gran número de factores y lo revisado junto a otros trabajos ${ }^{[11-14]}$ permite resumir lo siguiente:

- La capacidad de carga del carbón activado aumenta con el incremento de la concentración de cationes, en el siguiente orden:

$$
\mathrm{Ca}^{+2}>\mathrm{Mg}^{+2}>\mathrm{H}^{+}>\mathrm{Li}^{+}>\mathrm{Na}^{+}>\mathrm{K}^{+}
$$

pero, disminuye con el aumento de la concentración de aniones.

$$
\mathrm{CN}^{-}>\mathrm{S}^{-2}>\mathrm{SCN}^{-}>\mathrm{SO}_{3}^{2-}>\mathrm{OH}^{-}>\mathrm{Cl}^{-}>\mathrm{NO}_{3}^{-}
$$

- Cationes como $\mathrm{Ca}^{+2}, \mathrm{Mg}^{+2}$ y $\mathrm{Na}^{+}$son adsorbidos en presencia de $\mathrm{Au}(\mathrm{CN})_{2}^{-}$, no así en ausencia de $\mathrm{Au}(\mathrm{CN})_{2}^{-}$. Sin embargo, la concentración de cationes en el carbón es, generalmente, baja para un mecanismo de adsorción de par iónico.

- La adsorción de $\mathrm{Ca}^{+2}, \mathrm{Mg}^{+2}, \mathrm{Na}^{+}$y $\mathrm{K}^{+}$aumenta con el incremento del $\mathrm{pH}$ y disminuye $\mathrm{pa}$ ra $\mathrm{Au}(\mathrm{CN})_{2}^{-}$.

- La oxidación de grupos funcionales superficiales del carbón con cloro o ácido nítrico, produce una disminución en la capacidad de carga de $\mathrm{Au}(\mathrm{CN})_{2}^{-}$.

- La capacidad de carga del carbón aumenta con soluciones aireadas u oxigenadas.

Todas las formas anteriormente descritas como mecanismos son factibles que ocurran y, solamente, dependerán de las condiciones experimentales utilizadas. En términos cinéticos y gracias a numerosos trabajos experimentales se ha encontrado que cerca del 90 \% del área superficial del carbón, se encuentra en la categoría de microporo, siendo tal superficie inaccesible a las moléculas aurocianuradas, o solamente accesible después de una lenta y dificultosa difusión a través de los poros. La adsorción de aurocianuro en carbón activado es dependiente de muchos factores físicos y químicos, los cuales afectan tanto a la cinética de adsorción como a la capacidad de carga de aquel. La velocidad inicial de adsorción es rápida, siendo fundamental la hidrodinámica del reactor. Bajo estas condiciones, la velocidad es controlada por el transporte de masa en la película de líquido que rodea a las partículas de carbón, lo que implicaría la adsorción de las moléculas aurocianuradas en los sitios más accesibles, es decir, en los microporos y posibles mesoporos. Posteriormente, el oro sigue adsorbiéndose en forma lenta y dificultosa, lo que hace muy difícil obtener el verdadero equilibrio. Esto, requiere la difusión de las especies aurocianuradas a lo largo de los poros, dentro de la estructura del carbón, lo que configura un proceso, típicamente mucho más lento, que la difusión en capa límite, debido a la longitud y tortuosidad de los poros $^{[15-21]}$. La energía de activación para la adsorción de oro en carbón activado se ha estimado en un rango de $8-11 \mathrm{~kJ} / \mathrm{mol}$, lo cual está dentro de lo esperado para control por transporte de masa ${ }^{[22-25]}$.

Además, se han desarrollado diversos modelos matemáticos basados en la cinética de extracción de oro por carbón activado, los cuales han mejorado la comprensión de varios aspectos en el proceso de adsorción de oro. El modelo más simple asume que el proceso de adsorción de oro en carbón activado presenta una cinética de primer orden y desprecia los efectos del equilibrio carbón-disolución ${ }^{[26-28]}$. La constante cinética referida a una reacción de primer orden que tiene lugar en las etapas iniciales de la adsorción (lejos del equilibrio) se puede obtener desde:

$$
\frac{\mathrm{d}[\mathrm{Au}]_{\mathrm{s}}}{\mathrm{dt}}=-\mathrm{k} \cdot[\mathrm{Au}]_{\mathrm{s}}
$$

donde, $[\mathrm{Au}]_{\mathrm{s}}$ es la concentración de oro en la disolución a un tiempo t y k es la constante cinética de primer orden.

Pero, también, se han propuesto modelos más complejos que involucran tanto la transferencia de masa en la película líquida que rodea a las partículas de carbón, así como la difusión intrapartícula y la difusión superficial. A medida que se involucran mas resistencias a la transferencia de masa, los modelos cinéticos se vuelven más complejos ya sea en cuanto a su formulación como a su resolución. Sin embargo, representan, de una manera más fidedigna, lo que realmente ocurre durante la adsorción de oro en carbón activado y son extrapolables a tiempos mayores de adsorción, permitiendo, además, la determinación de parámetros cinéticos característicos. Aunque se han evaluado, también, modelos empíricos, estos permiten, solo, una limitada aplicación ${ }^{[29-34]}$.

En la actualidad, existe escasa información sobre estudios que reflejen la importancia de las propiedades físicas de los carbones activados y su implicación en el mecanismo y cinética de adsorción de oro, 
dentro del interior de la partícula del carbón estudiado. Estudios actuales predicen que la estructura grafítica del carbón activado es uno de los factores más importantes de proceso de adsorción de dicianuro de oro ${ }^{[35}$ y 36$]$. Sin embargo, han sido expuestas muy pocas consideraciones del rol de la estructura y propiedades físicas del carbón activado.

Dentro de la información actualizada, Seke et al. ${ }^{[37]}$ determinaron que existe una influencia de las condiciones superficiales del carbón activado en la adsorción del aurodicianuro, que involucra la distribución de tamaño de poro en la capacidad de adsorción del carbón: tres carbones activados fueron sometidos a pretratamiento a $850^{\circ} \mathrm{C}$ con gas de nitrógeno, observando que se incrementó la capacidad de adsorción de los carbones pretratados, sin embargo, no existió un cambio drástico en el volumen de los poros,antes y después de los tratamientos ${ }^{[1]}$.

Este trabajo tiene como objetivo estudiar la influencia de las propiedades físicas del carbón activado en el fenómeno de adsorción de oro desde medio cianuro tomando en cuenta, principalmente, las propiedades de superficie específica y distribución de tamaño de poros de los carbones estudiados y su relación con la velocidad de adsorción y cantidad de oro adsorbido.

\section{TRABAJO EXPERIMENTAL}

\subsection{Carbón activado}

Se realizaron pruebas de adsorción batch utilizando 2 tipos de carbón activado de diferentes características y procedencia denominados como Carbón A y Carbón B. En la tabla I se presenta la descripción general de los carbones activados utilizados en este estudio.

Tabla I. Descripción de los carbones activados en estudio

Table I. Description of the studied activated carbons

\begin{tabular}{lll}
\hline & Carbón A & Carbón B \\
\hline Forma & Granular & Granular \\
Materia Prima & Cáscaras de Coco & Cáscaras de Coco \\
Aplicación & Adsorción de Oro & $\begin{array}{l}\text { Purificación y } \\
\text { decoloración de } \\
\text { líquidos acuosos. } \\
\end{array}$ \\
& & Tratamiento de \\
& & aguas. \\
\hline
\end{tabular}

Previamente a su utilización, los carbones activados fueron acondicionados, para lo cual se realizó el siguiente procedimiento experimental:

- Se lavó el carbón activado con agua bidestilada y desionizada durante $30 \mathrm{~min}$, con agitación constante de $500 \mathrm{rpm}$.

- Se filtró la disolución con papel filtro $\mathrm{N}^{\circ} 2 \mathrm{pa}-$ ra remover toda partícula fina y el sólido remanente fue retirado y se colocó en una bandeja para posterior secado.

- Una vez finalizada la etapa de filtración, el carbón activado se secó en una mufla durante $1 \mathrm{~d}$, manteniéndolo a una temperatura dentro de un rango de 60 a $80^{\circ} \mathrm{C}$.

Los carbones activados se mantuvieron en un desecador con sílica gel durante todas las pruebas experimentales para mantener un nivel de humedad constante.

Los carbones fueron caracterizados determinando sus propiedades físicas y morfológicas. Para la caracterización morfológica se utilizó Microscopía Electrónica de Barrido y también se caracterizaron determinando la distribución de poros mediante el método BET, analizando la estructura interna de los carbones. Además, se tamizaron ambos carbones para determinar el diámetro promedio de las partículas, en base a la norma ASTM 2862 y, por último, se realizó un análisis de picnometría conducente a determinar la densidad de ambos carbones.

\subsection{Preparación de las disoluciones de oro y variables estudiadas}

Para la preparación de las disoluciones de oro se utilizó agua bidestilada y desionizada, y reactivos de grado analítico, como dicianurato de potasio $\left[\mathrm{K}\left(\mathrm{Au}(\mathrm{CN})_{2}\right)\right], \mathrm{CaCl}_{2}, \mathrm{NaCl}, \mathrm{KCl}, \mathrm{LiCl}$ y $\mathrm{KOH}$.

Los ensayos de adsorción batch se realizaron en un reactor de $500 \mathrm{ml}$ con agitación mecánica y a temperatura constante, controlada a través de un baño termorregulado. Se mantuvieron constantes los siguientes parámetros:

- Concentración inicial de oro : $6 \mathrm{ppm}$

$$
\begin{array}{ll}
\text { - Velocidad de agitación } & : 500(\mathrm{rpm}) \\
\text { - Tiempo de adsorción } & : 6(\mathrm{~h}) \\
\text { - Masa de carbón } & : 0,1(\mathrm{~g}) \\
\text { - } \mathrm{pH} & : 11 \\
\text { - Temperatura } & : 20^{\circ} \mathrm{C}
\end{array}
$$

Las variables estudiadas y sus rangos de experimentación se presentan en la tabla II. 
EFECTO DE LAS PROPIEDADES FÍSICAS DEL CARBÓN ACTIVADO EN LA ADSORCIÓN DE ORO DESDE MEDIO CIANURO EFFECT OF THE PHYSICAL PROPERITES OF ACTIVATED CARBON IN THE GOLD ADSORPTION FROM CYANIDE MEDIA

Tabla II. Variables y rangos de experimentación

Table II. Experimental variables and ranges

\begin{tabular}{|c|c|}
\hline Variable & Rango de experimentación \\
\hline $\begin{array}{l}\text { Tipo de carbón } \\
\text { Tipo y concentración } \\
\text { de cationes }\end{array}$ & $\begin{array}{l}\text { Carbón Ay Carbón B } \\
\mathrm{Na}^{+}, \mathrm{Li}^{+}, \mathrm{K}^{+} \text {y Ca }{ }^{+2} \\
(0,01-0,1-0,5)(\mathrm{M})\end{array}$ \\
\hline
\end{tabular}

\subsection{Metodología experimental}

La metodología experimental seguida para cada prueba fue la misma, variando, solo, las condiciones propias de cada caso. Las etapas desarrolladas fueron las siguientes:

- Se agregaron $400 \mathrm{ml}$ de la disolución acuosa de oro en el reactor que se dispuso en un baño termostático.

- Se ajustó el valor de pH de la disolución.

- Se controló la temperatura del baño termostático en forma paralela a los puntos anteriores.

- Se ajustó la velocidad del agitador mecánico a $500 \mathrm{rpm}$.

- Una vez acondicionada la disolución se incorporó el carbón activado.

- Se tomaron muestras de $5 \mathrm{ml}$ de la disolución a diferentes tiempos de muestreo.

- A las 6 h, se detuvo la experiencia.

Las muestras de disolución acuosa fueron enviadas a análisis químico por absorción atómica. El oro adsorbido en el carbón se determinó por balance de oro presente en las soluciones.

\section{RESULTADOS Y DISCUSION}

\subsection{Caracterización del carbón activado}

De acuerdo a lo descrito en el procedimiento experimental, el carbón activado fue caracterizado por diversas metodologías conducentes a obtener una imagen morfológica del mismo y la determinación de algunas propiedades físicas importantes.

En la tabla III se presenta un resumen de la caracterización de los carbones activados, considerada como representativa para las condiciones de adsorción. El porcentaje de macroporos, mesoporos y microporos obtenidos se relaciona con la clasificación de distribución de poros recomendada por IUPAC (International Union of Pure and Applied
Tabla III. Distribución de poros y propiedades físicas de los carbones activados

Table III. Pore distribution and physical properties of the activated carbons

\begin{tabular}{|c|c|c|}
\hline Propiedad & Carbón A & Carbón B \\
\hline Macroporos, \% & 41,04 & 63,21 \\
\hline Mesoporos, \% & 1,50 & 9,64 \\
\hline Microporos, \% & 57,46 & 27,16 \\
\hline $\begin{array}{l}\text { Diámetro promedio } \\
\text { de poros }(\mathrm{nm})\end{array}$ & 3,80 & 11,6 \\
\hline $\begin{array}{l}\text { Superficie Específica } \\
\left(\mathrm{m}^{2} / \mathrm{g}\right)\end{array}$ & 985,69 & 786,27 \\
\hline $\begin{array}{l}\text { Diámetro promedio } \\
\text { de partículas (mm) }\end{array}$ & 2,81 & 1,79 \\
\hline Densidad $\left(\mathrm{g} / \mathrm{cm}^{3}\right)$ & 1,35 & 1,18 \\
\hline
\end{tabular}

Chemistry), la cual dice que macroporos son aquellos que tienen diámetros mayores a $50 \mathrm{~nm}$, mesoporos, los que se sitúan entre 50 y $2 \mathrm{~nm}$ de diámetro y microporos, aquellos con diámetros inferiores a $2 \mathrm{~nm}$. De los datos ofrecidos se desprende que, para el carbón A, el 42,54 \% de los poros tiene una razón (diámetro de poro/diámetro del complejo) mayor a 5,2 y el 57,46\%, una razón inferior a 5,2. En cambio, para el carbón B, el 72,85 \% tienen una razón superior a 5,2 y, solo el $27,15 \%$, un valor inferior a 5,2. El elevado contenido de microporos del carbón A $(57,46 \%)$ le otorga un elevado valor de superficie específica de 985,69 $\mathrm{m}^{2} / \mathrm{g}$, con un diámetro promedio de partícula de 2,81 mm. En cambio, el carbón B tiene, solo, $786,27 \mathrm{~m}^{2} / \mathrm{g}$ de superficie específica y $1,79 \mathrm{~mm}$ de diámetro promedio de partícula.

Se realizó un análisis mediante microscopía electrónica para visualizar la superficie de los carbones activados de este estudio. Las imágenes capturadas mediante este equipo fueron obtenidas en una resolución de 500 y 2.000 aumentos. En las figuras 1 y 2 se presentan las micrografías electrónicas para el carbón A y B, respectivamente.

Al observar ambos carbones, en aumentos de 2000x se aprecian, claramente, diferencias a nivel superficial ya que el carbón $\mathrm{A}$, en la figura 1 , presenta una superficie externa formada aparentemente de placas planas con poros distinguibles a simple vista, compuestos, principalmente, de poros pequeños que le otorgan mayor área superficial comparado con el carbón B (Fig. 2). Este último, presenta una superficie cavernosa, no apreciándose, visiblemente, en su superficie externa, poros pequeños, por lo que los poros externos son, mayoritariamente, macroporos en su posición más expuesta a la adsorción. 

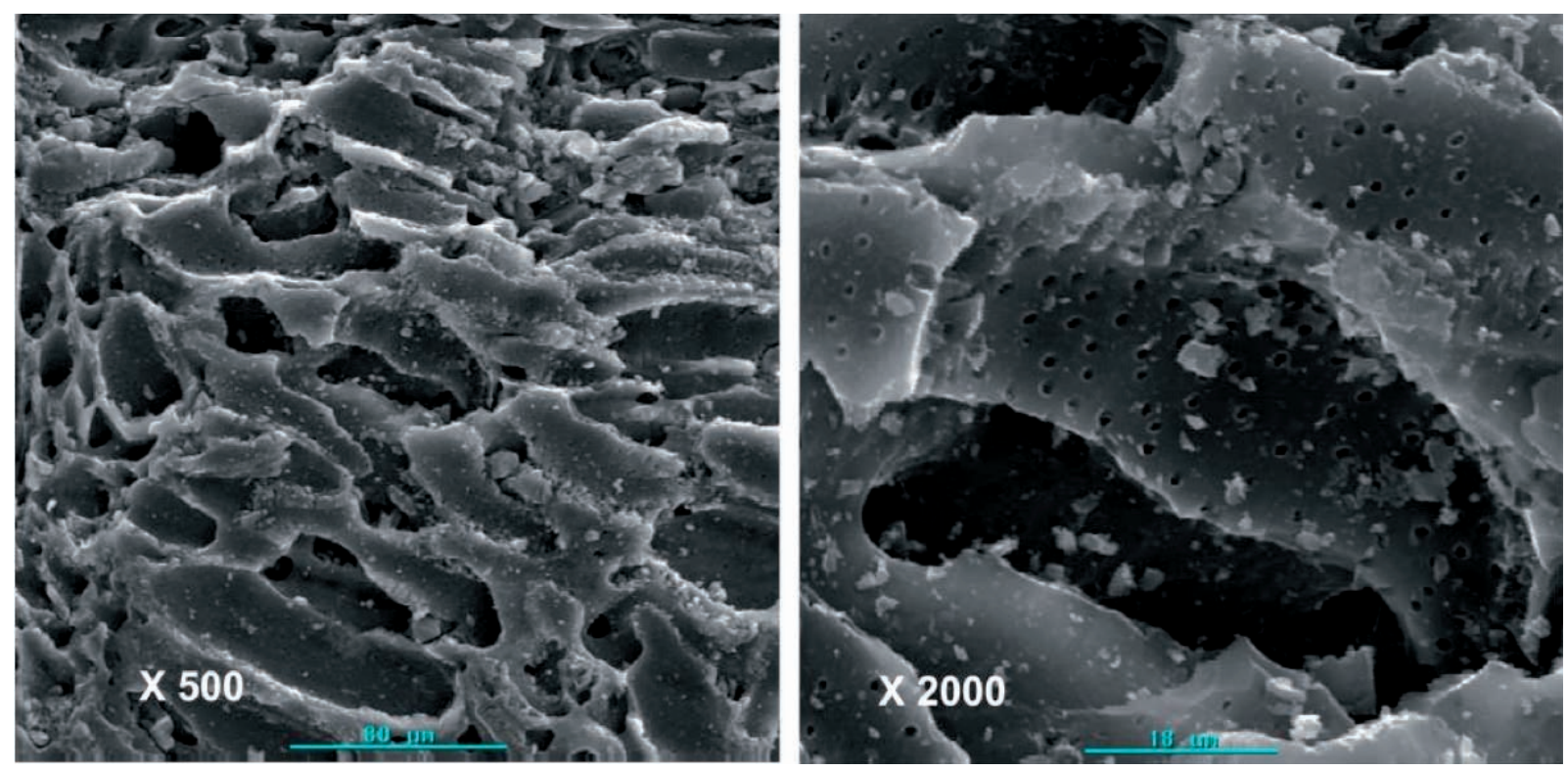

Figura 1. Micrografía electrónica para el carbón A.

Figure 1. Electronic micrography for carbon A.
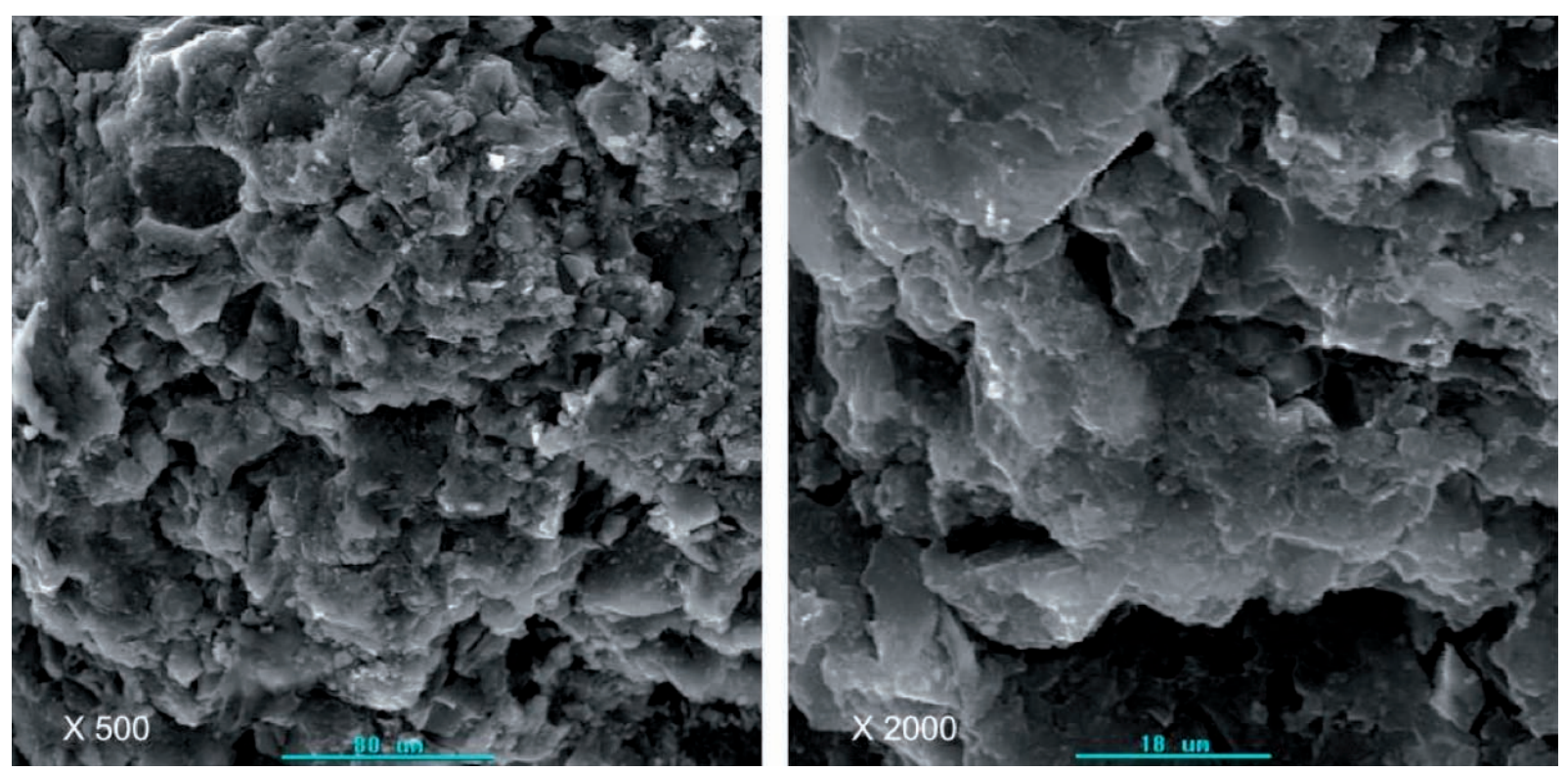

Figura 2. Micrografía electrónica para el carbón B.

Figure 2. Electronic micrography for carbon $B$.

\subsection{Efecto de cationes en la adsorción de oro}

Se evaluó el efecto de los cationes $\mathrm{Na}^{+}, \mathrm{Li}^{+}, \mathrm{K}^{+}$y $\mathrm{Ca}^{2+}$ en la adsorción de oro en el carbón activado, caracterizado como carbón A. Los resultados se presentan en las figuras 3-6.

Se encontró que al incrementar la concentración del ión $\mathrm{Na}^{+}$, de 0,01 a 0,5 M, la cantidad de oro adsorbido por $\mathrm{m}^{2}$ de superficie aumenta, considerablemente, a tiempos cortos. En la tabla IV se muestra la cantidad de oro adsorbido por $\mathrm{m}^{2}$ de superficie para 30 min de contacto carbón-disolución.

Cabe hacer notar que para ese intervalo de tiempo (0 a $30 \mathrm{~min}$ ) la velocidad de adsorción de oro es casi lineal, es decir, constante. La carga de oro con 


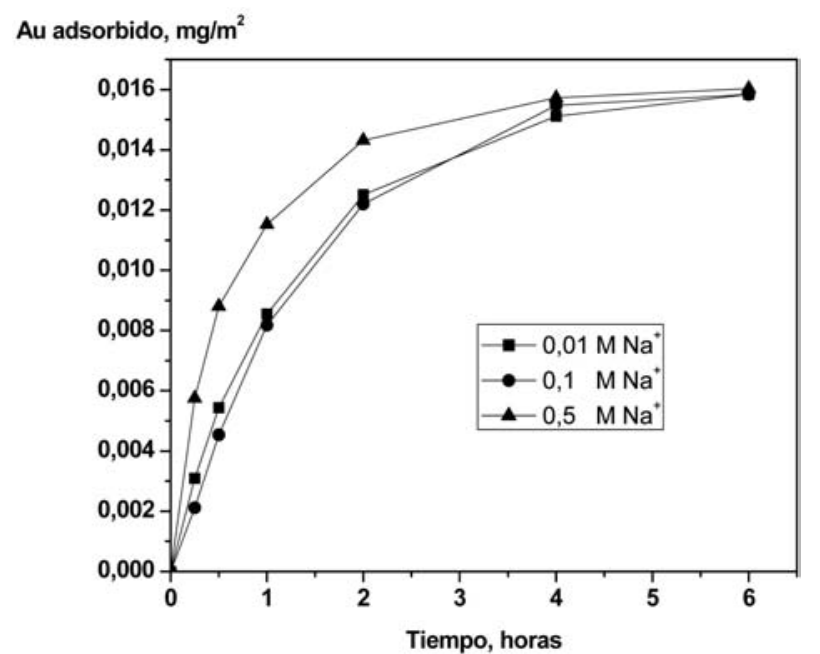

Figura 3. Efecto de la concentración de $\mathrm{Na}+$ en la adsorción de oro. Condición experimental: Carbón A; 0,1 g. de carbón activado; $\mathrm{pH}=11$; $\mathrm{T}=20^{\circ} \mathrm{C}$.

Figure 3. Effect of concentration of $\mathrm{Na}+$ in the gold adsorption. Experimental condition: Carbon A; $0,1 \mathrm{~g}$. of activated carbon; $\mathrm{pH}=11 ; \mathrm{T}=20^{\circ} \mathrm{C}$.

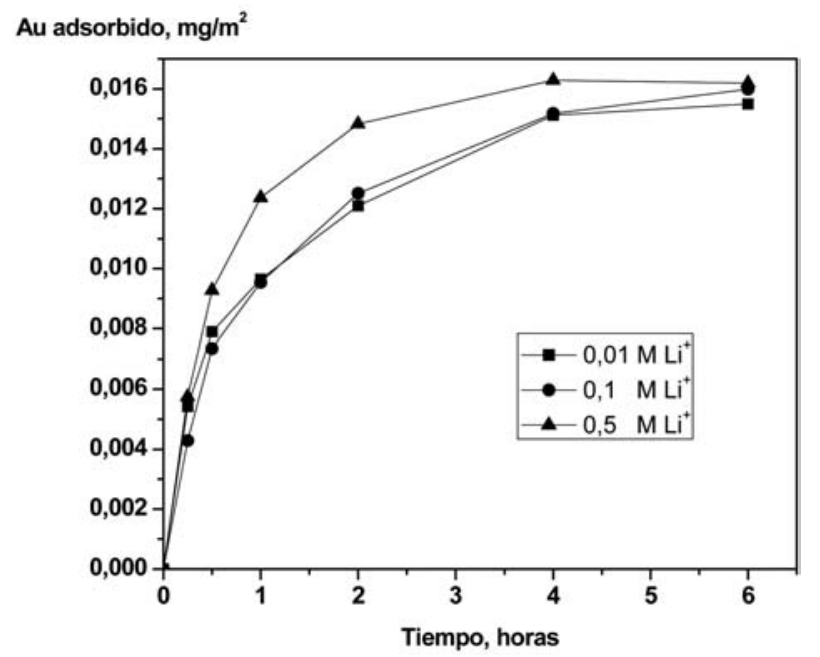

Figura 4. Efecto de la concentración de $\mathrm{Li}^{+}$en la adsorción de oro. Condición experimental: Carbón $\mathrm{A} ; 0,1 \mathrm{~g}$. de carbón activado; $\mathrm{pH}=11$; $\mathrm{T}=20^{\circ} \mathrm{C}$.

Figure 4. Effect of concentration of $\mathrm{Li}^{+}$in the gold adsorption. Experimental condition: Carbon A; $0,1 \mathrm{~g}$. of activated carbon; $\mathrm{pH}=11 ; T=20^{\circ} \mathrm{C}$.

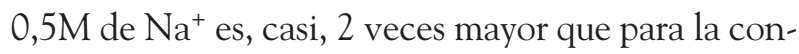
centración de $0,01 \mathrm{M}$ de $\mathrm{Na}^{+}$.

Para tiempos prolongados de contacto se alcanza un mismo valor de carga de oro para las diferentes

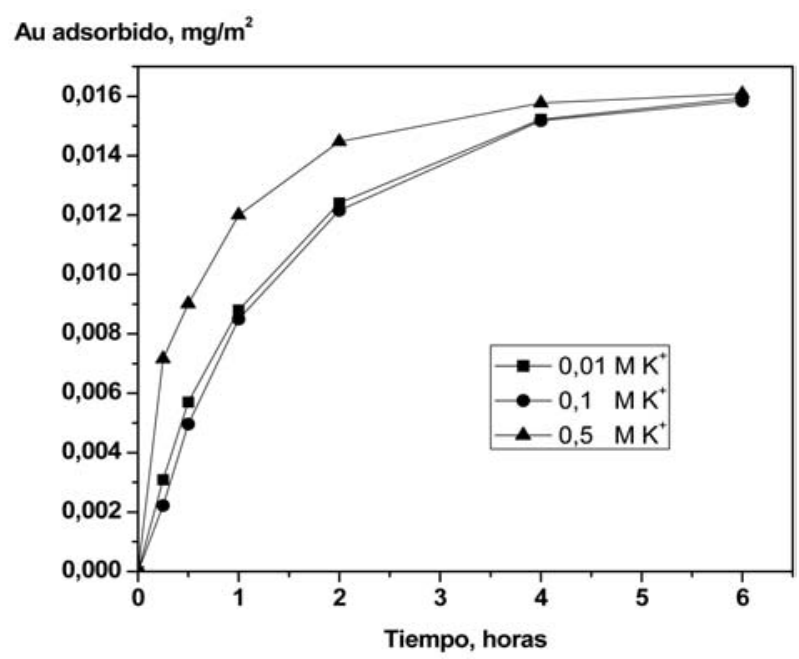

Figura 5. Efecto de la concentración de $\mathrm{K}^{+}$en la adsorción de oro. Condición experimental: Carbón A; 0,1 g. de carbón activado; $\mathrm{pH}=11$; $\mathrm{T}=20^{\circ} \mathrm{C}$.

Figure 5. Effect of concentration of $K^{+}$in the gold adsorption. Experimental condition: Carbon A; $0,1 \mathrm{~g}$. of activated carbon; $\mathrm{pH}=11 ; T=20^{\circ} \mathrm{C}$.

Au adsorbido, $\mathrm{mg} / \mathrm{m}^{2}$

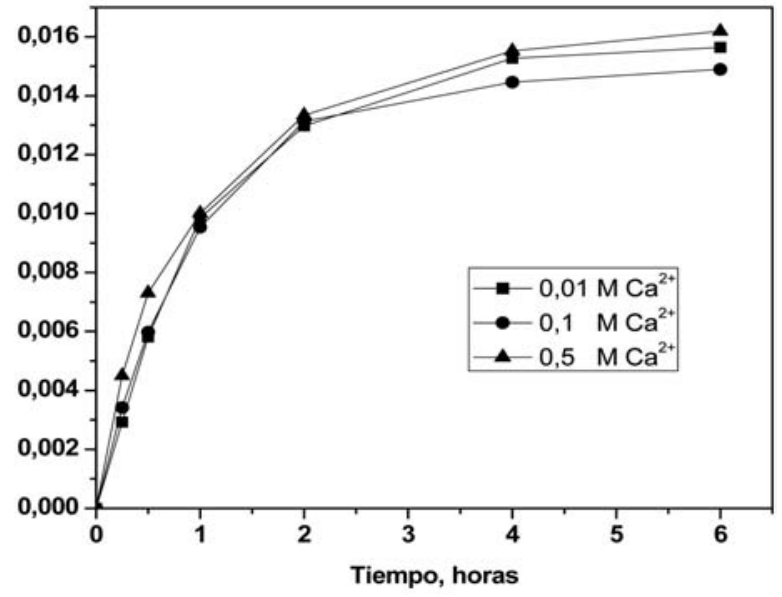

Figura 6. Efecto de la concentración de $\mathrm{Ca}^{2+}$ en la adsorción de oro. Condición experimental: Carbón $\mathrm{A} ; 0,1 \mathrm{~g}$. de carbón activado; $\mathrm{pH}=$ $11 ; \mathrm{T}^{\circ}=20^{\circ} \mathrm{C}$.

Figure 6. Effect of concentration of $\mathrm{Ca}^{2+}$ in the gold adsorption. Experimental condition: Carbon A; $0,1 \mathrm{~g}$ of activated carbon; $\mathrm{pH}=11 ; \mathrm{T}=20^{\circ} \mathrm{C}$.

concentraciones de $\mathrm{Na}^{+}$, debido a que ya no queda oro disuelto en la disolución acuosa. Durante todo el tiempo de contacto, el efecto de la concentración del ion $\mathrm{Na}^{+}$en el intervalo 0,01 a $0,1 \mathrm{M}$ fue pequeña y es 
Tabla IV. Carga de oro en el carbón A, a tiempos cortos de adsorción. Efecto de la concentración de sodio

Table IV. Load of gold on the carbon A, to short time of adsorption. Effect of sodium concentration

Carga de oro en el carbón, $\mathrm{mg}(\mathrm{Au}) / \mathrm{m}^{2}$ (carbón)

\begin{tabular}{cccc}
\hline Tiempo, $\min$ & $\mathbf{0 , 0 1 \mathrm { M } \mathrm { Na } +}$ & $\mathbf{0 , 1 \mathrm { M } \mathrm { Na } +}$ & $\mathbf{0 , 5 \mathrm { M } \mathrm { Na } +}$ \\
\hline 0 & 0 & 0 & 0 \\
15 & $4,8 \mathrm{E}-04$ & $6,7 \mathrm{E}-04$ & $12,1 \mathrm{E}-04$ \\
30 & $10,2 \mathrm{E}-04$ & $12,0 \mathrm{E}-04$ & $18,8 \mathrm{E}-04$
\end{tabular}

muy apreciable cuando se eleva la concentración a $0,5 \mathrm{M}$ de $\mathrm{Na}^{+}$.

El hecho de que la velocidad inicial de carga sea rápida ( 0 a $1 \mathrm{~h}$ de tiempo de contacto) se debe a que el proceso está controlado por la difusión del complejo $\mathrm{Au}(\mathrm{CN})_{2}^{-}$en la capa límite que rodea a la partícula de carbón, lo cual es concordante con lo encontrado por Fuertenau et. al. y Nicol et al., y a que la adsorción se produce en la superficie mas externa del carbón. Por otra parte, al aumentar la concentración de $\mathrm{Na}^{+}$, aumenta la cantidad de oro adsorbido, lo que, de alguna manera, confirma la predominancia del mecanismo de adsorción a la forma de par iónico. Posteriormente, la pendiente de las curvas disminuye debido a que comienza a agotarse el oro disuelto en la disolución acuosa y la difusión del complejo $\mathrm{Au}(\mathrm{CN})_{2}^{-}$ se hace más lenta con el tiempo. Para el caso del ión $\mathrm{Li}^{+}$y $\mathrm{K}^{+}$se observa el mismo comportamiento.

En el caso del catión $\mathrm{Ca}^{2+}$, no se aprecia diferencia importante al trabajar con las diferentes concentraciones de este elemento debido, probablemente, al efecto de la fuerza iónica, pues al ser éste un catión bivalente, la fuerza iónica de la disolución se cuadruplica, amentando el efecto de adsorción a la forma de par iónico, de acuerdo a lo propuesto por diferentes investigadores ${ }^{[6-10]}$.

Para estudiar el efecto del catión en la capacidad de carga de aurocianuro en el carbón activado se adoptó el coeficiente de distribución D, como una medida de la extracción, definido como:

$$
\mathrm{D}=\frac{\mathrm{mg} \mathrm{Au} / \mathrm{kg}}{\mathrm{mg} \mathrm{Au} / \mathrm{L}}=\frac{\mathrm{q}}{\mathrm{C}}
$$

donde, q es la carga de oro en el carbón en el equilibrio y C la concentración de oro residual en la disolución.
El efecto del tipo de catión en la extracción de oro se presenta en la figura 7. En ella, se se representa gráficamente la forma logarítmica del coeficiente de distribución en función del radio iónico del catión particular. Se observa la tendencia al aumento de la solubilidad de la especie aurocianurada a medida que aumenta el radio iónico, por ello, la estabilidad del complejo aurocianuro de potasio se ve favorecida, reflejándose, esto, en una disminución en la extracción de oro. La mayor extracción de oro en presencia de $\mathrm{Ca}^{2+}$ se debe a un efecto de fuerza iónica, en vez de al tamaño de radio iónico ya que un ión divalente produce una fuerza iónica mayor, a igual concentración. Es destacable mencionar que Tsuchida et al. ${ }^{[17]}$ encontraron que solo la carga del ión era importante y no el tamaño, pero sus resultados fueron obtenidos a fuerzas iónicas mucho más bajas.

\subsection{Comparación del efecto de cationes en carbón A y B}

En este punto se estudió la influencia de diferentes cationes en la adsorción de oro, utilizando los dos carbones en estudio, es decir, carbón A, cuya superficie específica es de $985 \mathrm{~m}^{2} / \mathrm{g}$ con $57 \%$ de microporos y carbón B, con $786 \mathrm{~m}^{2} / \mathrm{g}$, de superficie específica y $27 \%$ de microporos. En la figura 8 se presenta el efecto de la presencia de cationes para los dos carbones en estudio.

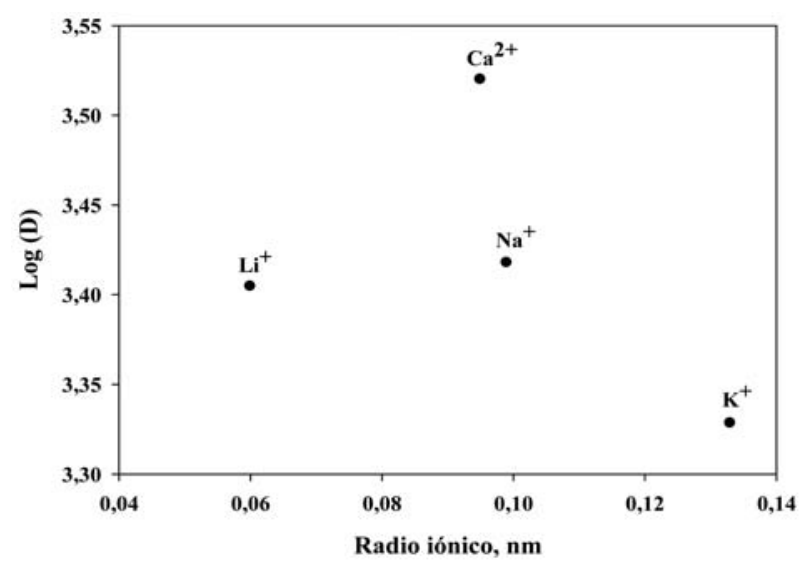

Figura 7. Coeficiente de Distribución de oro en función del radio iónico de los cationes en estudio. Condición experimental: Carbón A; 0,1 g. de carbón activado; $0,1 \mathrm{M}$ de cationes; $\mathrm{pH}=11$; $\mathrm{T}=20^{\circ} \mathrm{C}$.

Figure 7. Gold distribution coefficient in function of ionic radius of the cations in study. Experimental condition: Carbon $A ; 0,1 \mathrm{~g}$. of activated carbon; $0,1 \mathrm{M}$ of cations; $p H=11 ; T=20^{\circ} \mathrm{C}$. 

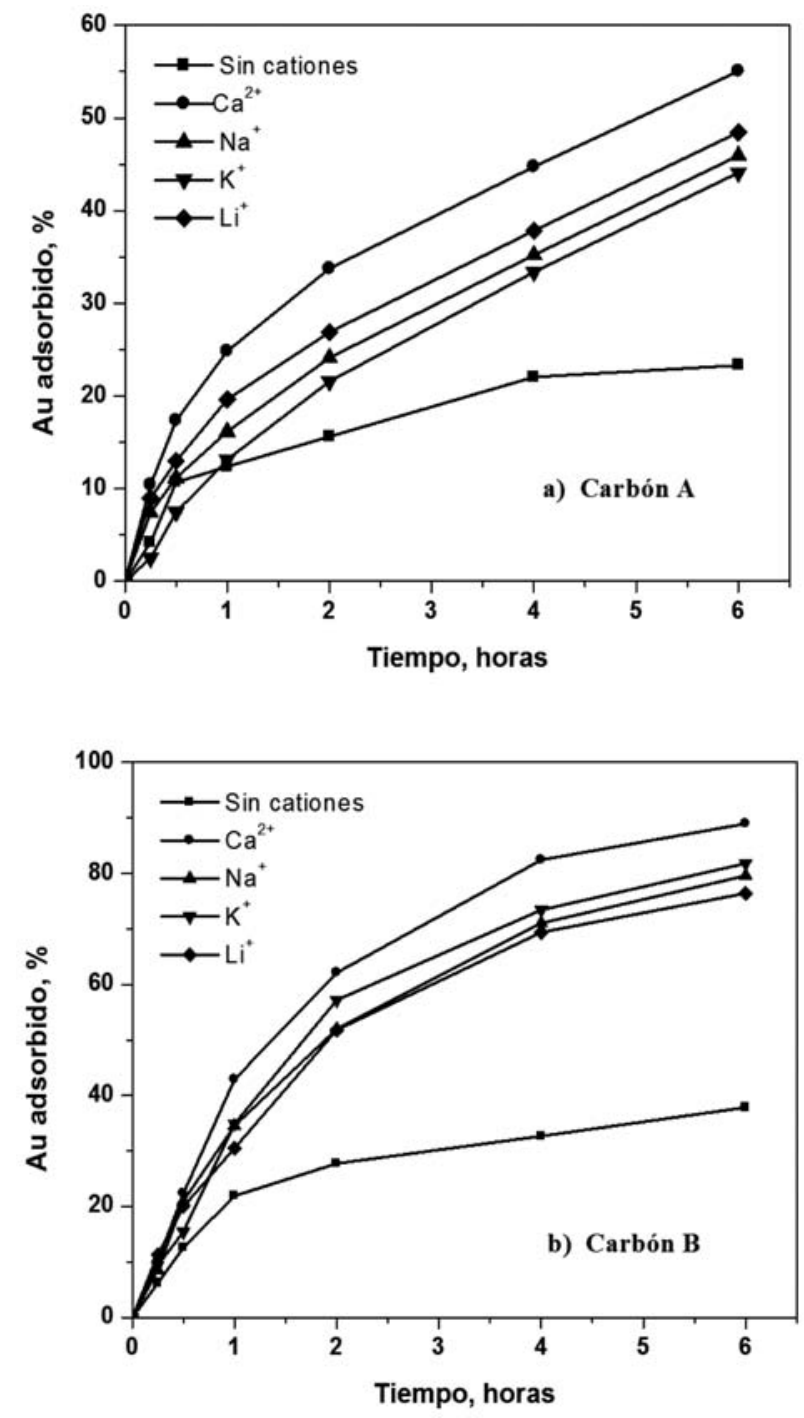

Figura 8. Efecto de la presencia de cationes en la adsorción de oro. Condición experimental: Carbón $\mathrm{A}$ y $\mathrm{B} ; 0,1 \mathrm{~g}$. de carbón activado; $\mathrm{pH}=$ $11 ; \mathrm{T}=20^{\circ} \mathrm{C}$, concentración de cationes $0,1 \mathrm{M}$.

Figure 8. Effect of the presence of cations in the gold adsorption. Experimental condition: carbon $A$ and $B ; 0,1 \mathrm{~g}$. of activated carbon; $\mathrm{pH}=11 ; \mathrm{T}$ $=20^{\circ} \mathrm{C}$, cations concentration $0,1 \mathrm{M}$.

La velocidad de adsorción de oro utilizando el carbón A sigue el orden: $\mathrm{Ca}^{2+}>\mathrm{Li}^{+}>\mathrm{Na}^{+}>\mathrm{K}^{+}>\sin$ cationes. Para el caso del carbón B, el efecto de los cationes en la adsorción de oro sigue el orden: $\mathrm{Ca}^{2+}>$ $\mathrm{K}^{+}>\mathrm{Na}^{+}>\mathrm{Li}^{+}>$sin cationes.

En todo el rango de tiempo destaca la mayor adsorción de oro para el carbón B, de menor superficie específica y menor porcentaje de microporos, para todos los iones estudiados. Después de $6 \mathrm{~h}$ de contacto, la adsorción se movió en el rango de $72 \%$ usando $\mathrm{Li}^{+}$y $88 \%$ con $\mathrm{Ca}^{2+}$; en cambio, para el carbón A, para el mismo tiempo, se alcanzó un $45 \%$ de adsorción usando $\mathrm{Na}^{+}$y $55 \%$ con $\mathrm{Ca}^{2+}$.

De acuerdo a lo propuesto por Fuerstenau et al..$^{[18]}$, la velocidad de adsorción de oro en carbón activado es controlada por transferencia de masa en la película líquida que rodea a la partícula de carbón, despreciando los efectos del equilibrio, lo que hace que el proceso sea irreversible y aplicable solo para cargas bajas de oro en el carbón. Luego, la expresión de un modelo cinético de primer orden puede representar lo anteriormente planteado:

$$
\mathrm{C}_{\mathrm{Au}}=\mathrm{C}_{\mathrm{Au}}^{\mathrm{o}} \cdot \mathrm{e}^{-\frac{\mathrm{M}}{\mathrm{V}} \cdot \mathrm{k} \cdot \mathrm{t}}
$$

donde, $\mathrm{C}_{\mathrm{Au}}$ es la concentración de oro en disolución a un tiempo, $\mathrm{t}, \mathrm{C}_{\mathrm{Au}}{ }^{\circ}$, la concentración inicial de oro disuelto, M, masa de carbón, V, volumen de disolución, $\mathrm{k}$, constante cinética y $\mathrm{t}$, tiempo. Se aplicó este modelo de acuerdo a lo mostrado en la figura 9, obteniéndose los valores de las constantes cinéticas mostradas en la tabla V.

La mayor adsorción de oro, en todos los casos, con el carbón B, puede ser atribuida a dos hechos: en primer lugar, este carbón tiene un diámetro medio de partícula de $1,79 \mathrm{~mm}$, valor bastante menor al del carbón A que tiene 2,81 mm, lo cual para una misma masa de carbón le proporciona una mayor superficie externa, que se torna importante a bajas cargas de oro. Por otra parte, el mayor contenido de macroporos del carbón B (63,2 \%) en relación al A (41,04 \%), permite una mayor facilidad de transporte del oro y de los cationes al interior de la partícula, todo lo cual se ve reflejado en los mayores valores de las constantes cinéticas obtenidas para el carbón B.

En ambos casos de la figura 8, se puede concluir que se produjo una mayor cantidad de oro adsorbido en presencia de cationes, lo cual se fundamenta en el mecanismo de adsorción a la forma de par iónico.

El estudio posteriormente fue continuado usando ión $\mathrm{Ca}^{2+}$, debido al mayor efecto que ejerce en la adsorción (en relación a los otros iones) y además, al ser el catión más abundante en las aguas industriales.

En figuras 10 y 11 se observan los picos característicos de los iones calcio y oro, detectados mediante la técnica de Fluorescencia de Rayos X, para los carbones A y B, respectivamente, una vez finalizadas las experiencias de adsorción. La presencia de oro y calcio adsorbidos confirman que el mecanismo predominante de adsorción es del tipo par iónico, que se representa de la siguiente forma:

$$
\mathrm{Ca}^{2+}(\mathrm{s})+2 \mathrm{Au}(\mathrm{CN})_{2}^{-}(\mathrm{s})=\mathrm{Ca}\left[\mathrm{Au}(\mathrm{CN})_{2}\right]_{2}(\mathrm{ads})
$$




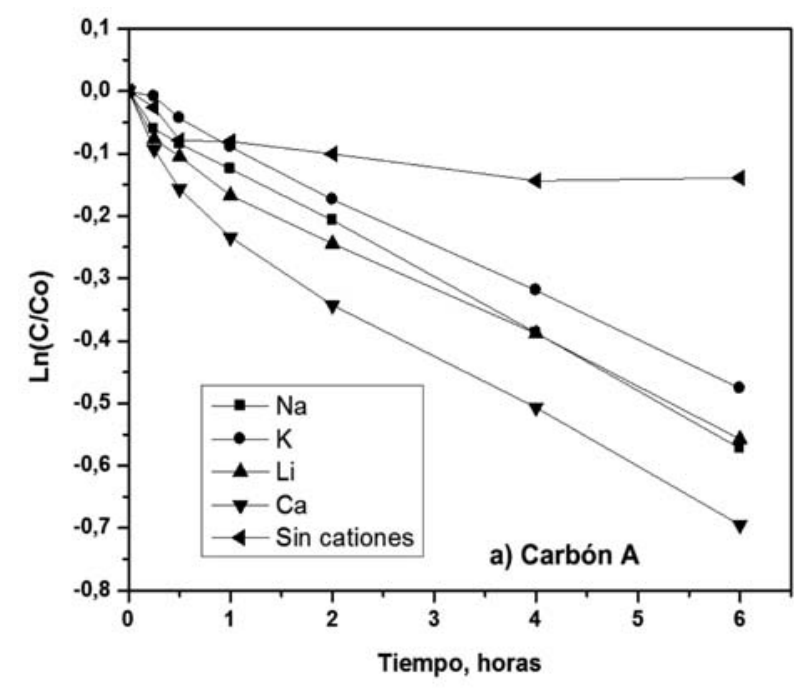

Tabla V. Constantes cinéticas para los carbones $\mathrm{A}$ y $\mathrm{B}$ para $0,1 \mathrm{M}$ de los cationes en estudio

Table V. Kinetic constants for the carbons $A$ and $B$ for $0,1 M$ of the cations in study

\begin{tabular}{lrr}
\hline \multicolumn{1}{c}{ Catión } & $\begin{array}{c}\text { Carbón A } \\
\mathbf{k}(\mathbf{1} / \mathbf{h})\end{array}$ & $\begin{array}{r}\text { Carbón B } \\
\mathbf{k}(\mathbf{1} / \mathbf{h})\end{array}$ \\
\hline Sin cationes & 127 & 221 \\
$\mathrm{Na}^{+}$ & 292 & 802 \\
$\mathrm{Li}^{+}$ & 295 & 742 \\
$\mathrm{~K}^{+}$ & 241 & 867 \\
$\mathrm{Ca}^{2+}$ & 378 & 1.132 \\
\hline
\end{tabular}
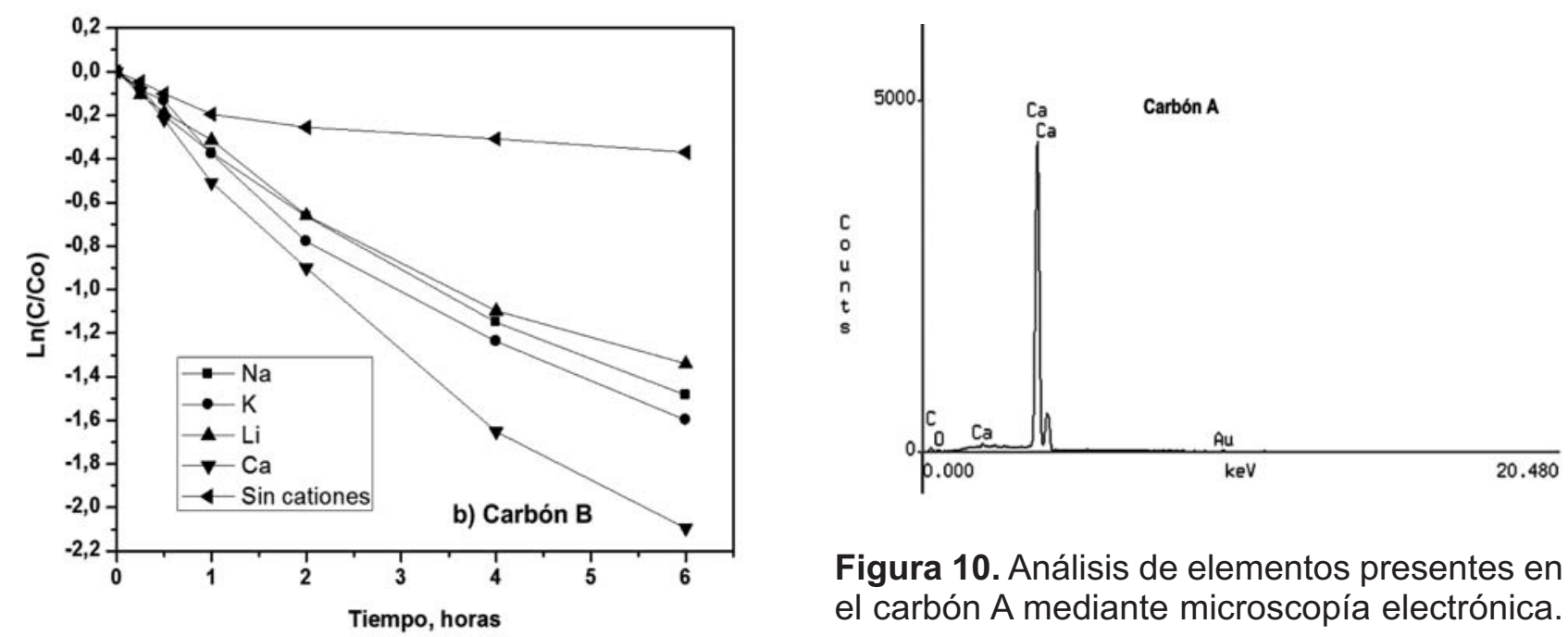

Figura 9. Aplicación del modelo de primer orden para la determinación de la constante cinética k. Condición experimental: Carbón A y B; $0,1 \mathrm{~g}$. de carbón activado; $0,1 \mathrm{M}$ de cationes; 6 horas de adsorción; $\mathrm{pH}=11 ; \mathrm{T}=20^{\circ} \mathrm{C}$.

Figure 9. Application of first order model for the determination of kinetic constant $k$. Experimental condition: Carbon A and B; 0,1 G. of activated carbon; $0,1 \mathrm{M}$ of cations; 6 hours of adsorption; $\mathrm{pH}=11 ; \mathrm{T}=20^{\circ} \mathrm{C}$.

\subsection{Efecto de la distribución de tamaño de poros}

Para observar el efecto de la distribución de tamaño de poros en la adsorción de oro se realizaron ensayos, a iguales condiciones experimentales, para los dos carbones en estudio. En la figura 12 se muestra el efecto de esta variable para ambos carbones.

Figura 10. Análisis de elementos presentes en el carbón A mediante microscopía electrónica.

Figure 10. Analysis of elements present in carbon A by electron microscopy.

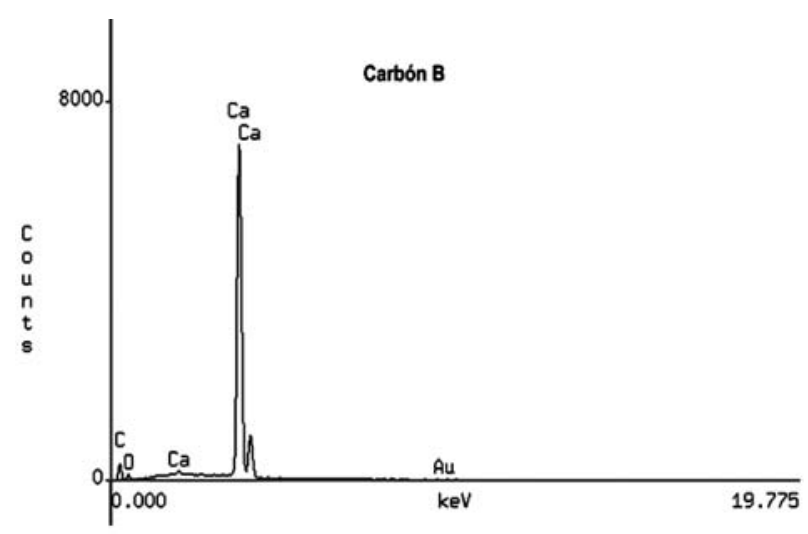

Figura 11. Análisis de elementos presentes en el carbón B mediante microscopía electrónica.

Figure 11. Analysis of elements present in carbon $B$ by electron microscopy. 
EFECTO DE LAS PROPIEDADES FÍSICAS DEL CARBÓN ACTIVADO EN LA ADSORCIÓN DE ORO DESDE MEDIO CIANURO EFFECT OF THE PHYSICAL PROPERITES OF ACTIVATED CARBON IN THE GOLD ADSORPTION FROM CYANIDE MEDIA

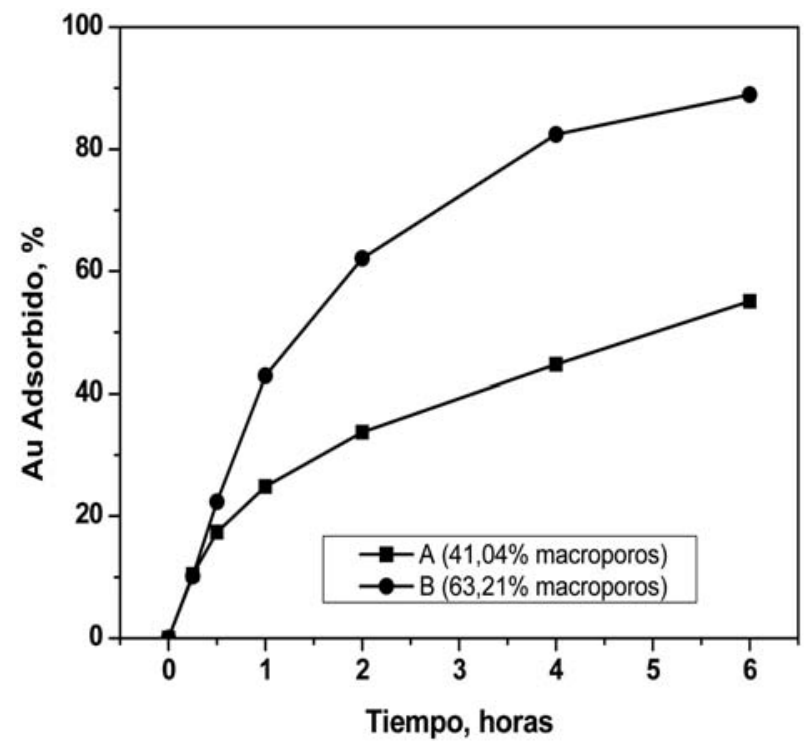

Figura 12. Efecto del \% de macroporos en la adsorción de oro. Condición experimental: 0,1 g. de carbón activado; $\mathrm{pH}=11, \mathrm{~T}=20^{\circ} \mathrm{C} ; 0,1$ $\mathrm{M} \mathrm{Ca}^{+2}$.

Figure 12. Effect of percentage of macropores in the gold adsorption. Experimental conditions: $0,1 \mathrm{~g}$. of activated carbon; $\mathrm{pH}=11 ; \mathrm{T}=20^{\circ} \mathrm{C}$; $0,1 \mathrm{MCa}^{2+}$.

Se puede observar que el carbón B, que posee mayor cantidad de macroporos $(63,21 \%)$, adsorbe mayor cantidad de oro que el carbón A (41,04 \% macroporos), después de $6 \mathrm{~h}$ de contacto carbóndisolución.

En los primeros $15 \mathrm{~min}$, la adsorción de ambos carbones fue similar, pero después, el carbón B presentó una mayor capacidad de adsorción que prevaleció hasta las $6 \mathrm{~h}$ de proceso. En cambio, el carbón $\mathrm{A}$ adsorbe una menor cantidad de oro en todo el rango experimental.

De acuerdo a los valores de las constantes cinéticas encontradas y mostradas en la tabla $\mathrm{V}$, el valor de $\mathrm{k}$ para el carbón $\mathrm{B}$ cuando se trabajó con $\mathrm{Ca}^{2+}$ es $1.132 \mathrm{l} / \mathrm{h}$ y, para el carbón A, $378 \mathrm{l} / \mathrm{h}$, es decir, tres veces mayor para el carbón $B$. En la tabla VI se muestran las razones de las constantes cinéticas para todos los cationes estudiados, es decir, $\mathrm{k}_{\mathrm{B}} / \mathrm{k}_{\mathrm{A}}$, donde, $\mathrm{k}_{\mathrm{A}}$ es la constante cinética de adsorción de oro para el carbón $A$ y $k_{B}$, para el carbón B.

De la tabla VI se puede deducir que la razón de las constantes cinéticas para los distintos cationes en estudio fluctúa en un valor de 3 , lo que permite afirmar que la velocidad de adsorción de oro en carbón B es 3 veces mayor que con el carbón A, para situaciones lejanas del equilibrio.
Tabla VI. Relación entre las constantes cinéticas de los carbones estudiados
Table VI. Relation between the kinetic constants of the studied carbons

\begin{tabular}{ll}
\hline Catión & kB/kA \\
\hline $\mathrm{Na}^{+}$ & 2,75 \\
$\mathrm{Li}^{+}$ & 2,52 \\
$\mathrm{~K}^{+}$ & 3,60 \\
$\mathrm{Ca}^{2+}$ & 3,00 \\
\hline
\end{tabular}

Lo anteriormente descrito indica que, inicialmente, la adsorción del complejo $\mathrm{Au}(\mathrm{CN})_{2}^{-}$se produce en la superficie mas externa del carbón, no afectando la diferencia de porosidades (parte lineal inicial de las curvas de la figura 12), fenómeno que estaría controlado por la difusión del complejo en el líquido inmediatamente adyacente a la superficie externa del carbón. Posteriormente, el complejo de oro tiene que difundir hacia el interior de la partícula de carbón, razón por la cual la cantidad de oro adsorbido a tiempos más prolongados es menor para el carbón A que para el $\mathrm{B}$ ya que el fenómeno está influenciado por el tamaño de los poros a través de los cuales debe transportarse el complejo $\mathrm{Au}(\mathrm{CN})_{2}^{-}$.

\subsection{Efecto del diámetro promedio de poros}

En este punto se relaciona la adsorción de oro en presencia de diferentes cationes con el tamaño promedio de poros de los carbones. En el caso del carbón $\mathrm{A}$, el tamaño promedio de sus poros es de 3,8 nm y, en el del carbón B, de 11,6 nm, lo cual representa un $305 \%$ del diámetro promedio mayor del carbón $\mathrm{B}$ en relación al A.

En la figura 13 se muestra la cantidad de oro adsorbido en $\mathrm{g}(\mathrm{Au}) / \mathrm{kg}$ (carbón), tras 6 h de contacto carbón-disolución, para los dos carbones en estudio, en presencia de diferentes tipos de iones.

La cantidad de oro adsorbido es mayor, en todos los casos, con el carbón B (11,6 nm de diámetro promedio de poros), lo cual es un reflejo de la mayor superficie externa que posee dicho carbón y de la mayor facilidad que tiene el complejo $\mathrm{Au}(\mathrm{CN})_{2}^{-}$, de transportarse al interior de las partículas.

Con el carbón B se adsorbió entre 13,5 y $16 \mathrm{~g}$ $(\mathrm{Au}) / \mathrm{kg}$ (carbón), en cambio, con el carbón A, 


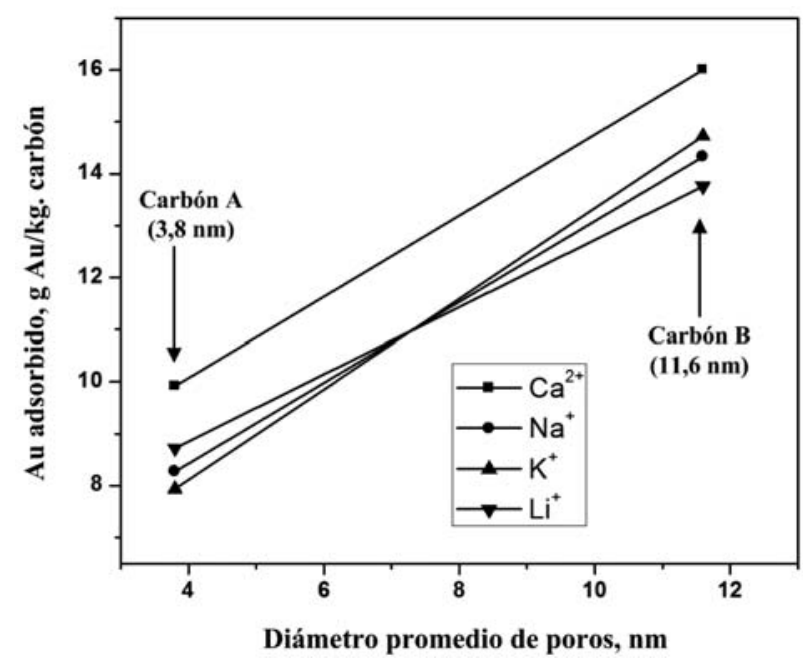

Figura 13. Efecto del diámetro promedio de poros en la carga de oro en el carbón en presencia de diferentes cationes. Condición experimental: $0,1 \mathrm{~g}$. de carbón activado; $\mathrm{pH}=11 ; \mathrm{T}=20^{\circ} \mathrm{C}$.

Figure 13. Effect of average pore diameter in the gold adsorption in presence of different cations. Experimental conditions: $0,1 \mathrm{~g}$. of activated carbon; $\mathrm{pH}=11 ; \mathrm{T}=20^{\circ} \mathrm{C}$.

entre 8 y $10 \mathrm{~g}(\mathrm{Au}) / \mathrm{kg}$ (carbón), lo cual representa un $64 \%$, en promedio, de mayor carga del carbón $B$ en relación al $A$.

\subsection{Efecto del área superficial}

En principio, cabría pensar que a mayor superficie específica mejores serian las características, como adsorbente, del carbón activado, puesto que también se debería tener un mayor número de sitios disponibles para adsorber el complejo $\mathrm{Au}(\mathrm{CN})_{2}^{-}$. Sin embargo, esto no siempre se cumple ya que se debe tener en cuenta el posible "efecto de tamiz molecular". Así, dependiendo del tamaño de los complejos que serán adsorbidos puede suceder que parte de la superficie específica no sea accesible a dichas moléculas o que, en su defecto, sea de difícil acceso.

En la figura 14 se presenta la carga de oro por unidad de superficie de ambos carbones en estudio, en función del tiempo. Se observa que el carbón B $\left(786,27 \mathrm{~m}^{2} / \mathrm{g}\right)$ presenta una mayor carga de oro por unidad de área superficial, alcanzando a duplicar la carga de oro en relación al carbón A $(985,69$ m²/g) al final de las 6 h de contacto carbón-disolución.

La mayor carga de oro por unidad de área superficial obtenida en el carbón B está asociada, directamente, a la distribución de tamaño de poros de di-

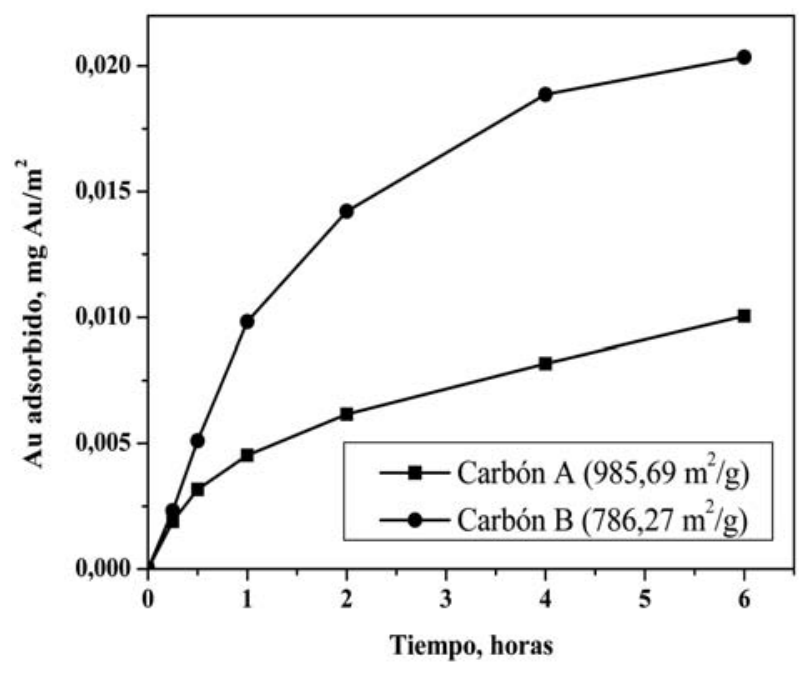

Figura 14. Efecto del área superficial del carbón en la adsorción de oro. Condición experimental: $0,1 \mathrm{~g}$. de carbón activado; $\mathrm{pH}=11 ; \mathrm{T}=$ $20{ }^{\circ} \mathrm{C} ; 0,1 \mathrm{M} \mathrm{Ca}^{+2}$.

Figure 14. Effect of carbon surface area in the gold adsorption. Experimental conditions: $0,1 \mathrm{~g}$. of activated carbon; $\mathrm{pH}=11 ; \mathrm{T}=20^{\circ} \mathrm{C} ; 0,1 \mathrm{M}$ $\mathrm{Ca}^{2+}$.

cho carbón, con un tamaño promedio de 11,6 nm en relación al carbón $\mathrm{A}$, con 3,8 nm, lo cual, también, se traduce en, aproximadamente, $200 \mathrm{~m}^{2}$ menos de área específica que posee el carbón B.

Todo lo anteriormente planteado produce una mayor facilidad de transporte del complejo $\mathrm{Au}(\mathrm{CN})_{2}^{-}$ al interior de la partícula de carbón, ya sea por difusión a través de la disolución que llena los poros y por migración superficial a través de las paredes de los poros hacia el interior de la partícula.

\section{CONCLUSIONES}

- La presencia de cationes favorece el proceso de adsorción de oro en medio cianuro, especialmente, los de mayor valencia, lo cual puede ser atribuido al mecanismo de adsorción vía par iónico.

- Una mayor cantidad de macroporos en un carbón activado produce una mayor adsorción de oro a tiempos cortos de contacto carbón-disolución.

- El aumento el diámetro promedio de poros en un carbón activado, aumenta la carga de oro por unidad de superficie específica.

- Con una menor área superficial del carbón activado se obtuvo una mayor carga de oro a tiempos cortos o lejos del equilibrio. 
EFECTO DE LAS PROPIEDADES FÍSICAS DEL CARBÓN ACTIVADO EN LA ADSORCIÓN DE ORO DESDE MEDIO CIANURO EFFECT OF THE PHYSICAL PROPERITES OF ACTIVATED CARBON IN THE GOLD ADSORPTION FROM CYANIDE MEDIA

- Las propiedades físicas de un carbón activado son un factor importante en el proceso de adsorción de oro, en términos de velocidad de adsorción y cantidad de oro adsorbido. El carbón B, con $786 \mathrm{~m}^{2} / \mathrm{g}$ de superficie especifica, alcanzó una mayor carga por unidad de superficie $(0,02 \mathrm{mg}$ $\left.\mathrm{Au} / \mathrm{m}^{2}\right)$, en relación al carbón A que posee 985 $\mathrm{m}^{2} / \mathrm{g}$, el cual alcanzó una carga de 0,01 mg Au/ $\mathrm{m}^{2}$, después de $6 \mathrm{~h}$ de contacto.

- Los valores experimentales de experiencias cinéticas se ajustaron perfectamente a un modelo lineal de primer orden, encontrándose que los valores de $\mathrm{k}_{\mathrm{B}}$ fueron alrededor de 3 veces mayores a los de $\mathrm{k}_{\mathrm{A}}$, para los diferentes cationes en estudio.

\section{Agradecimientos}

Los autores agradecen a la Comisión Nacional de Investigación Científica y Tecnológica (CONICYT) por el patrocinio otorgado para la realización de este trabajo científico a través del proyecto Fondecyt №1070091.

\section{REFERENCIAS}

[1] R. Merello y G. Zárate, Minerales 42 (1985) 25-39.

[2] C. Fleming, Hydrometallurgy 30 (1992) 127-162.

[3] J. Marsden y L. House, The Chemistry of Gold Extraction, 2aㅡ ed. SME, 2006, pp. 260-266.

[4] G. Mc Dougall y R. Hancock, Gold Bulletin 14 (1981) 138-153.

[5] G. Mc Dougall, J. S. Afr. I. Min. Metall. 91 (1991) 109-120.

[6] R. J. Davidson, J. S. Afr. I. Min. Metall. 75 (1974) 67-74.

[7] G. Mc Dougall, R. Hancock, M. Nicol, O. Wellington y R. Copperthwaite, J. S. Afr. I. Min. Metall. 80 (1980) 344-356.

[8] V. López, C. Moreno, J. Rivera y L. Radovic, Carbon 41 (2002), 2.020-2.022.

[9] M. Adams y C. Fleming, Metall. Mater. Trans. B. 20 (1989) 315-325.

[10] M. Adams, Hydrometallurgy 25 (1990) 171-184.

[11] M. Adams, J. S. Afr. I. Min. Metall. 90 (1990) 37-44.

[12] M. Adams, J. Friedl y F. Wagner, Hydrometallurgy 37 (1995) 33-45.
[13] K. Rees, J. Van Deventer, Hydrometallurgy 58 (2000) 151-167.

[14] M. Yalcin y A. Arol, Hydrometallurgy 63 (2002) 201-206.

[15] E. Cho y C. Pitt, Metall. Mater. Trans. B. 10 (1979) 165-169.

[16] E. Cho, S. Dixon y C. Pitt, Metall. Mater. Trans. B. 10 (1979) 185-189.

[17] N. Tsuchida y D. Muir, Metall. Mater. Trans. B. 17 (1986) 529-533.

[18] M. Fuerstenau, C. Nebo, J. Kelso y R. Zaragoza, Miner. Metall. Process. 4 (1987) 177-181.

[19] P. Van der Merwe y J. Van Deventer, Chem. Eng. Comm. 65 (1988) 121-138.

[20] W. Jones y H. Linge, Hydrometallurgy 22 (1989) 231-238.

[21] R. Jordi, B. Young y A. Bryson, Chem. Eng. Comm. 102 (1991) 127-147.

[22] F. Petersen y J. Van Deventer, Chem. Eng. Science. 46 (1991) 3.053-3.065.

[23] J. Van Deventer y P. Van Der Merwe, Metall. Mater. Trans. B. 24 (1993) 433-440.

[24] W. Yapu, M. Segarra, M. Fernandez y F. Espiell, Metall. Mater. Trans. B. 25 (1994) 185-191.

[25] N. Vegter y R. Sanderbergh, J. S. Afr. I. Min. Metall. 96 (1996) 109-118.

[26] J. Van Deventer, Chem. Eng. Comm. 44 (1986) 257-274.

[27] G. Mc Dougall, M. Adams y R. Hancock, Hydrometallurgy 18 (1987) 125-138.

[28] M. Adams, G. Mc Dougall y R. Hancock, Hydrometallurgy 18 (1987) 139-154.

[29] M. Adams, G. Mc Dougall y R. Hancock, Hydrometallurgy 19 (1987) 95-115.

[30] L. Woollacott, W. Stange y R. King, J. S. Afr. I. Min. Metall. 90 (1990) 275-282.

[31] J. Le Roux, A. Bryson y B. Young, J. S. Afr. I. Min. Metall. 91 (1991) 95-103.

[32] N. Vegter, Hydrometallurgy 30 (1992) 229-242.

[33] F. Ahmed, B. Young y A. Bryson, Hydrometallurgy 30 (1992) 257-275.

[34] N. Syna y M. Valix, Miner. Eng. 16 (2003) 421427.

[35] P. Sibrell y J. Miller, Miner. Metall. Proc. 9 (1992) 189-195.

[36] A. Ibrado y D. Fuersteanau, Hydrometallurgy 30 (1992) 243-256.

[37] M. Seke, R. Sandenbergh y N. Vegter, Miner. Eng. 13 (2000) 527-540. 